\title{
Neuromodulation Therapy with Vagus Nerve Stimulation for Intractable Epilepsy: A 2-Year Efficacy Analysis Study in Patients under 12 Years of Age
}

\author{
Suresh Gurbani, ${ }^{1,2}$ Sirichai Chayasirisobhon, ${ }^{1}$ Leslie Cahan, ${ }^{1}$ SooHo Choi, ${ }^{1}$ \\ Bruce Enos, ${ }^{1}$ Jane Hwang, ${ }^{1}$ Meei Lin, ${ }^{1}$ and Jeffrey Schweitzer ${ }^{1}$ \\ ${ }^{1}$ Comprehensive Epilepsy Program, Southern California Permanente Medical Group, CA, USA \\ ${ }^{2}$ Department of Neurology, Kaiser Permanente Medical Center, Suite No. 208, 3460 E. La Palma Avenue, Anaheim, CA 92806, USA
}

Correspondence should be addressed to Suresh Gurbani; suresh.g.gurbani@kp.org

Received 28 October 2015; Revised 13 January 2016; Accepted 13 January 2016

Academic Editor: József Janszky

Copyright (c) 2016 Suresh Gurbani et al. This is an open access article distributed under the Creative Commons Attribution License, which permits unrestricted use, distribution, and reproduction in any medium, provided the original work is properly cited.

To study the efficacy of vagus nerve stimulation (VNS) therapy as an adjunctive treatment for intractable epilepsy in patients under 12 years of age, we analyzed 2-year postimplant data of 35 consecutive patients. Of the 35 patients, 18 (51.4\%) at 6 months, 18 (51.4\%) at 12 months, and $21(60.1 \%)$ at 24 months showed $\geq 50 \%$ reduction in seizure frequency (responders). Although incremental seizure freedom was noted, no patient remained seizure-free throughout the 3 study periods. Partial response ( $\geq 50 \%$ seizure reduction in 2 or less study periods) was seen in $8(22.9 \%)$ patients. Twelve patients (34.3\%) were nonresponders. Out of 29 patients with primary generalized epilepsy, 20 (68.9\%) and, out of 6 patients with focal epilepsy, 3 (50\%) had $\geq 50 \%$ seizure control in at least one study period. No major complications or side effects requiring discontinuation of VNS therapy were encountered. We conclude that (1) patients with intractable primary generalized epilepsy respond better to VNS therapy, (2) cumulative effect of neuromodulation with improving responder rate to seizure freedom with continuation of VNS therapy is noted, and (3) VNS therapy is safe and is well tolerated in children receiving implant under 12 years of age.

\section{Introduction}

Neuromodulation therapies are nonpharmacotherapeutic options for patients with drug resistant epilepsy who are not candidates for resective epilepsy surgery. In 1997, the US Food and Drug Administration (FDA) approved vagus nerve stimulation (VNS) implant as adjunctive therapy for reducing the frequency of seizures in patients $>12$ years of age with partial-onset seizures refractory to antiepileptic drugs (AEDs) [1].

Initial studies with randomized controlled trials reporting on the efficacy of VNS involved rather short followup duration ( 3 months to 3.5 months) and $\geq 50 \%$ seizure reduction ranged from $23.4 \%$ to $39 \%$ of the patients [2-5]. Since 1999, several studies have reported long-term followup ranging from 6 months to 10 years with $\geq 50 \%$ seizure reduction observed in $35 \%$ to $63.8 \%$ of the patients [6-22].
Literature search identified 16 studies regarding the efficacy of VNS in children [17, 18, 23-36]. However, most of these studies did not truly reflect the efficacy of VNS therapy in children $<12$ years old as they included older patients as well. Ten of the 16 studies included subjects through 18 years of age, and one each included subjects up to 19, 20, 21, and 25 years of age. One study of 11 patients with tuberous sclerosis had a mean age of 14 years with a range from 2 to 35 years [23]. In this study, we report long-term (2 years) observation on the efficacy and the safety of VNS in epileptic patients $<12$ years of age.

\section{Methods}

Ours is a prospectively collected data analysis retrospective study. All patients with epilepsy being treated at our center 
maintain daily seizure diary which is entered in the electronic database during follow-up visits. All patients with recalcitrant epilepsy undergo long-term video EEG monitoring and neuroimaging studies including MRI brain examination and are presented at Kaiser multidisciplinary epilepsy surgery case conference to discuss alternate nonpharmacotherapeutic treatment options. A total of 160 patients with drug resistant epilepsy (failed at least 3 AEDs at adequate doses appropriate for the type of epilepsy) who were not candidates for resective surgery received VNS implant from September 1998 to December 2011, 35 of whom were $<12$ years of age at the time of implant. VNS device from Cyberonics was implanted by our neurosurgeons who had received the required training.

To allow wound healing, the VNS implant was not activated until one week postoperatively. Output current was gradually increased in $0.25 \mathrm{~mA}$ increments once per week at six weekly visits, then at six subsequent biweekly visits, and then at each of three monthly visits to the clinic. After an informed decision by the parents, type of VNS cycling (standard versus rapid) and parameters were selected by the treating pediatric epileptologist. For standard cycling, the signal on-time was $\geq 30$ seconds and signal off-time ranged from 3 to 5 minutes. For rapid cycling, signal on-time was $\leq 21$ seconds and signal off-time ranged from 0.2 to 1.8 minutes. Rapid cycling was initiated with the parameters of 7 seconds on-time and 0.2 minutes off-time. Output current was adjusted depending on the patient's tolerance to the electrical stimulation and seizure control. Maximum output current used was $3.0 \mathrm{~mA}$ for rapid cycling and $3.5 \mathrm{~mA}$ for standard cycling. Ongoing AED regimen (dosing regimen and if needed AED) was adjusted as clinically warranted.

The efficacy of VNS therapy was analyzed by comparing the mean seizure frequency (prior 8-week period) at baseline (at VNS implant) to that at 6-month, 12-month, and 24month postimplant study points. We defined the efficacy of VNS therapy as follows: (1) responders: $\geq 50 \%$ reduction of seizures in all three study periods, (2) partial responders: $\geq 50 \%$ reduction of seizures in 2 or less study periods, and (3) nonresponders: $<50 \%$ response and/or worsening seizure control in all 3 study periods. Efficacy of standard versus rapid cycling therapy parameters was also studied. We analyzed the efficacy of VNS therapy according to the type of epilepsies as well. We also assessed for postoperative adverse events, side effects, and tolerability of both the surgical implantation procedure and the VNS device.

\section{Results}

Thirty-five patients (23 males, 12 females) with age ranging from 5 years to 12 years (mean age, $7.79 \pm 2.65$ years) met the selection criteria. Clinical characteristics for the patients at baseline are summarized in Table 1. Mean age of onset of epilepsy was $1.25 \pm 1.55$ years. Mean duration of epilepsy before the VNS implant was $6.67 \pm 2.95$ years. Mean number of AEDs at baseline was 2.5. Mean output current setting was $1.9 \pm 0.7 \mathrm{~mA}$ at 6 months, $2.3 \pm 0.7 \mathrm{~mA}$ at 12 months, and $2.5 \pm 0.7 \mathrm{~mA}$ at 24 months. Of the 35 patients, $18(51.4 \%)$ at 6
TABLE 1: Clinical data of patients at baseline.

\begin{tabular}{lc}
\hline (A) Epilepsy and seizure types & \\
Generalized tonic-clonic & 9 patients \\
Absence atypical & 5 patients \\
Tonic & 3 patients \\
Myoclonic & 1 patient \\
Atonic & 1 patient \\
Focal with secondary generalized tonic-clonic & 4 patients \\
Focal with dyscognitive features & 2 patients \\
Mixed & 10 patients \\
(B) Etiology & \\
Lennox-Gastaut syndrome & 13 patients \\
Postencephalitis & 5 patients \\
Cortical dysgenesis & 4 patients \\
Postanoxic encephalopathy & 3 patients \\
Idiopathic & 3 patients \\
Tuberous sclerosis & 3 patients \\
Chromosomal abnormality & 2 patients \\
Stroke & 2 patients \\
\hline
\end{tabular}

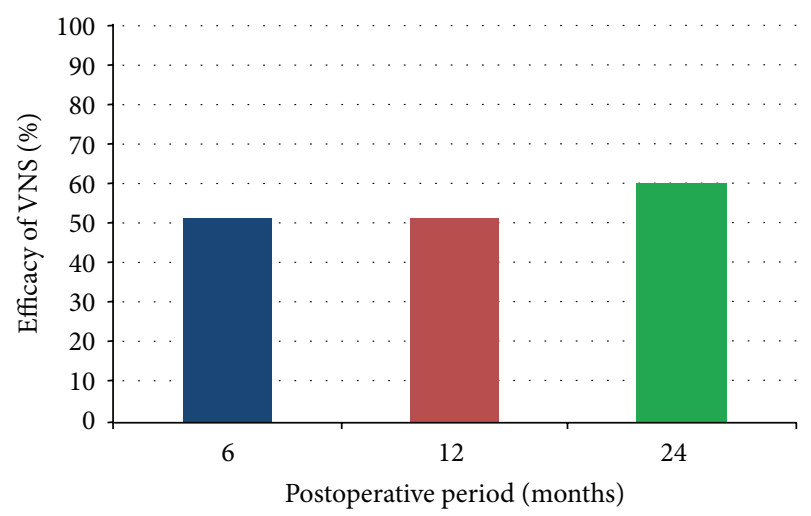

FIGURE 1: Efficacy of VNS ( $\geq 50 \%$ reduction in seizure frequency) at 3 study periods.

months, $18(51.4 \%)$ at 12 months, and $21(60.1 \%)$ at 24 months showed $\geq 50 \%$ reduction in seizure frequency (Figure 1 ).

Although, among the responders, a complete (100\%) seizure control was seen in 4 of 18 patients $(22.2 \%)$ at 6 month, 5 of 18 patients $(27.8 \%)$ at 12 -month, and 7 of 21 patients $(33.3 \%)$ at 24 -month follow-up period, no single patient remained seizure-free throughout the 3 study periods. A total of $15(42.9 \%)$ patients had $\geq 50 \%$ reduction in seizure frequency in all three periods and a partial response was seen in $8(22.8 \%)$ more patients. Twelve patients (34.3\%) showed no clinically significant benefit in all three periods (Table 2).

For the 22 patients who were treated with rapid cycle of VNS, the output current ranged from $0.75 \mathrm{~mA}$ to $3 \mathrm{~mA}$ and pulse width from 125 microseconds to 250 microseconds, with signal on-time set at 7 seconds to 14 seconds and signal off-time ranging from 0.5 minutes to 1.1 minutes. Seizure frequency reduction of $\geq 50 \%$ was seen in 11 of 22 patients (50\%) at 6-month, 11 of 22 patients (50\%) at 12-month, and 
TABLE 2: The efficacy of VNS with $\geq 50 \%$ reduction of seizures for all patients at 6 months, 12 months, and 24 months.

\begin{tabular}{lcc}
\hline Study period & $\begin{array}{c}\text { Number of } \\
\text { patients }\end{array}$ & Percent \\
\hline At 6 months, 12 months, and 24 months & 15 & 42.9 \\
At 6 months & 2 & 5.7 \\
At 6 months and 24 months & 1 & 2.8 \\
At 12 months and 24 months & 3 & 8.6 \\
At 24 months & 2 & 5.7 \\
No response & 12 & 34.3 \\
\hline
\end{tabular}

12 of 22 patients (54.5\%) at 24-month follow-up period. The remaining 13 patients were treated with the standard cycle of VNS with output current ranging from $0.75 \mathrm{~mA}$ to $3.5 \mathrm{~mA}$ and pulse width from 250 microseconds to 500 microseconds, with signal on-time of 30 seconds and signal off-time from 3 minutes to 5 minutes. Seizure frequency reduction of $\geq 50 \%$ was seen in 7 of 13 patients $(53.8 \%)$ at 6-month, 6 of 13 patients (46.2\%) at 12-month, and 9 of 13 patients $(69.2 \%)$ at 24 -month follow-up period.

The efficacy of VNS according to the etiologies of epilepsy is shown in Table 3 and according to the seizure types in Table 4. During the study period 25 patients had a single type of seizures, 6 patients had 2 types of seizures, and 4 patients had 3 types of seizures. Out of 35 patients, 29 $(82.9 \%)$ had primary generalized epilepsy and $6(17.1 \%)$ had focal epilepsy. Twenty patients $(68.9 \%)$ with primary generalized epilepsy and 3 patients (50\%) with focal epilepsy had $\geq 50 \%$ reduction in seizure frequency. Best responders were patients with primary generalized epilepsy with tonicclonic seizures followed by primary generalized epilepsy with atypical absence seizures.

Cough and pharyngeal paresthesia commonly occurred during initial application or ramming up of output current. These adverse events were successfully managed by adjusting the parameters. No side effects necessitating discontinuation of VNS therapy were encountered.

\section{Discussion}

VNS therapy has been approved as adjunctive treatment for drug resistant focal epilepsy in patients $>12$ years of age [1]. However, as drug resistant epilepsies in pediatric population are also an unconquered challenge despite availability of second and third generation AEDs and as the epilepsy treatment goal remains seizure freedom, VNS therapy has been used in patients $<12$ years of age as well. Many researchers have reported the efficacy of VNS for treatment of epilepsy in children [17, 18, 23-36]. Among these 16 studies, ten studies included subjects through 18 years of age, and one each included subjects up to $19,20,21$, and 25 years of age. One study of 11 patients with tuberous sclerosis had a mean age of 14 years with a range from 2 to 35 years [23]. Our study reports the efficacy and safety of VNS therapy in a group of 35 epileptic patients $<12$ years of age.
Similar to the observations of prior VNS studies, with increasing duration of VNS therapy, a trend towards improving responder rate and seizure freedom was noted in our study as well [37-39]. The exact mechanism for the improving efficacy of VNS with increasing duration of therapy is not fully understood. Chronic therapeutic response to VNS therapy is highly correlated with bilateral thalamic increases in synaptic activity [40]. During chronic VNS therapy, brain excitatory amino acid neurotransmitter levels are reduced and inhibitory neurotransmitter levels are increased but no direct relationship to seizure control has been noted [40].

Optimal parameter settings for VNS therapy are not yet well defined. In current study, both rapid and standard cycle settings of VNS parameters were used. Patients who were treated with rapid cycling showed $\geq 50 \%$ reduction of seizure frequency in all three periods while those treated with standard cycling showed $\geq 50 \%$ reduction of seizure at 6-month and 24-month periods. Both rapid cycling and standard cycling demonstrated the cumulative seizure response to VNS therapy at 24-month period. The comparison of the efficacy between rapid cycling and standard cycling was not conclusive. Our previous study in 39 patients (age ranging from 5 to 72 years) demonstrated a trend towards greater seizure frequency reduction in patients with rapid cycle than standard cycle parameters. It also showed that when compared to adult patients, the response to rapid cycle in pediatric patients was greater [41]. However, a 2year follow-up study has reported greater overall seizure frequency reduction with the standard cycle than the rapid cycle [12]. Other studies did not show any difference in responder rate with either cycle $[24,29]$. More research with larger population is recommended to study this further.

In our study, VNS therapy was effective in both focal epilepsy and some types of generalized epilepsy. VNS therapy has been reported to be effective in patients with LennoxGastaut syndrome $[27,29,30]$ and tuberous sclerosis complex $[36,37]$. In our study, VNS therapy was effective in achieving $\geq 50 \%$ reduction in seizure in patients with LennoxGastaut syndrome, encephalitis, cortical dysgenesis, perinatal encephalopathy, and tuberous sclerosis complex. Best responders were patients with primary generalized epilepsy with tonic-clonic seizures followed by primary generalized epilepsy with atypical absence seizures. Small sample size did not permit statistical analysis by seizure types and etiologies.

Our patients tolerated the VNS implantation well. There was no serious wound infection requiring explantation. No major complications or side effects requiring discontinuation of VNS therapy occurred during the 2-year study period.

Patients included in this study were offered VNS as an adjunctive treatment modality to ongoing antiepilepsy medication regimen and not after failing most available antiepilepsy medications. Therefore, change in the dosing regimen and, if needed, in the antiepilepsy medications used (which is a limitation of this study) was done per the choice of the treating physician. As many more AED choices (second and third generation AEDs) are available now, with a small study subject size it was not possible to comment on synergism of any specific AED mechanism of action being responsible for the seizure control noted after VNS implant. 
TABLE 3: The efficacy of VNS with $\geq 50 \%$ reduction of seizures according to the etiologies of epilepsy.

\begin{tabular}{lccc}
\hline Etiology (patients) & Responders & Partial responders & Nonresponders \\
\hline Lennox-Gastaut syndrome (13) & $38.5 \%$ & $23.0 \%$ & $38.5 \%$ \\
Postencephalitis (5) & $20.0 \%$ & $40.0 \%$ & $40.0 \%$ \\
Cortical dysgenesis (4) & $75.0 \%$ & $0 \%$ & $25.0 \%$ \\
Postanoxic encephalopathy (3) & $33.3 \%$ & $0 \%$ & $66.7 \%$ \\
Perinatal encephalopathy (3) & $66.7 \%$ & $0 \%$ & $33.3 \%$ \\
Tuberous sclerosis (3) & $50.0 \%$ & $50.0 \%$ & $0 \%$ \\
Chromosomal abnormality (2) & $50.0 \%$ & $0 \%$ & $50.0 \%$ \\
Stroke (2) & $50.0 \%$ & $50.0 \%$ & $0 \%$ \\
\hline
\end{tabular}

TABLE 4: Number of patients with $\geq 50 \%$ reduction of seizures according to the seizure types during 3 study periods.

\begin{tabular}{lccc}
\hline Seizure type (\# at baseline) & 6 months & 12 months & 24 months \\
\hline Generalized tonic-clonic (16) & 10 & 11 & 11 \\
Absence atypical (8) & 6 & 5 & 5 \\
Tonic (8) & 4 & 4 & 4 \\
Myoclonic (4) & 3 & 1 & 0 \\
Atonic (5) & 2 & 3 & 2 \\
Focal with generalized & 0 & 2 & 2 \\
tonic-clonic (4) & & & \\
Focal with dyscognitive & 1 & 1 & 1 \\
features (2) & & & \\
\hline
\end{tabular}

Many studies have reported lack of improvement in responder rate after failing 2 appropriate AEDs [1]. All the patients in the current study had failed at least 3 AEDs in adequate doses appropriate for the type of epilepsy before VNS therapy was initiated. Therefore, it can be safely assumed that in these patients maximum response to AEDs had already been attained and reduction in seizure frequency noted after the implant can be attributed to the VNS therapy. On the other hand, the patient's desire to decrease the dose and reduce the number of AEDs after VNS implantation may have resulted in a negative impact on the responder rate.

In conclusion, neuromodulation with VNS therapy can be used successfully as an adjunctive treatment for patients $<12$ years of age with both focal and generalized drug resistant epilepsies.

The cumulative seizure response to VNS therapy necessitates long-term efficacy analysis studies. Due to availability of third generation AEDs it is unethical to design a prospective double blind research study with unchanged AED regimen to further define the findings of this study. Therefore, our retrospective data analysis study has limited but definite scientific contribution.

\section{Conflict of Interests}

The authors declare that there is no conflict of interests regarding the publication of this paper.

\section{References}

[1] Cyberonics, VNS Therapy Products Manuals and Safety Alerts: Part1-Introduction-Indications, Warnings and Precautions, Cyberonics, Houston, Tex, USA, 2012.

[2] M. C. Salinsky, B. M. Uthman, R. K. Ristanovic, J. F. Wernicke, and W. B. Tarver, "Vagus nerve stimulation for the treatment of medically intractable seizures: results of a 1-year open-extension trial," Archives of Neurology, vol. 53, no. 11, pp. 1176-1180, 1996.

[3] G. W. Hornig, J. V. Murphy, G. Schallert, and C. Tilton, "Left vagus nerve stimulation in children with refractory epilepsy: an update," Southern Medical Journal, vol. 90, no. 5, pp. 484-488, 1997.

[4] J. V. Murphy, G. Hornig, and G. Schallert, "Left vagal nerve stimulation in children with refractory epilepsy: preliminary observations," Archives of Neurology, vol. 52, no. 9, pp. 886-889, 1995.

[5] E. Ben-Menachem, R. Mañon-Espaillat, R. Ristanovic et al., "Vagus nerve stimulation for treatment of partial seizures: 1. A controlled study of effect on seizures. First International Vagus Nerve Stimulation Study Group," Epilepsia, vol. 35, no. 3, pp. 616-626, 1994.

[6] C. M. DeGiorgio, S. C. Schachter, A. Handforth et al., "Prospective long-term study of vagus nerve stimulation for the treatment of refractory seizures," Epilepsia, vol. 41, no. 9, pp. 11951200,2000

[7] M. Frost, J. Gates, S. L. Helmers et al., "Vagus nerve stimulation in children with refractory seizures associated with LennoxGastaut syndrome," Epilepsia, vol. 42, no. 9, pp. 1148-1152, 2001.

[8] S. Chayasirisobhon, W. V. Chayasirisobhon, S. Koulouris, and S. Gurbani, "Vagus nerve stimulation in drug-resistant epilepsy," Acta Neurologica Taiwanica, vol. 12, pp. 123-129, 2003.

[9] D. R. Labar, "Antiepileptic drug use during the first 12 months of vagus nerve stimulation therapy: a registry study," Neurology, vol. 59, no. 6, supplement 4, pp. S38-S43, 2002.

[10] A. P. Amar, C. M. DeGiorgio, W. B. Tarver, and M. L. Apuzzo, "Long-term multicenter experience with vagus nerve stimulation for intractable partial seizures: results of the XE5 trial," Stereotactic and Functional Neurosurgery, vol. 73, no. 1-4, pp. 104-108, 1999.

[11] E. Ben-Menachem, K. Hellström, C. Waldton, and L. E. Augustinsson, "Evaluation of refractory epilepsy treated with vagus nerve stimulation for up to 5 years," Neurology, vol. 52, no. 6, pp. 1265-1267, 1999.

[12] J. Scherrmann, C. Hoppe, T. Kral, J. Schramm, and C. E. Elger, "Vagus nerve stimulation: clinical experience in a large patient 
series," Journal of Clinical Neurophysiology, vol. 18, no. 5, pp. 408-414, 2001.

[13] J. V. Murphy, R. Torkelson, I. Dowler, S. Simon, and S. Hudson, "Vagal nerve stimulation in refractory epilepsy: the first 100 patients receiving vagal nerve stimulation at a pediatric epilepsy center," Archives of Pediatrics and Adolescent Medicine, vol. 157, no. 6, pp. 560-564, 2003.

[14] B. M. Uthman, A. M. Reichl, J. C. Dean et al., "Effectiveness of vagus nerve stimulation in epilepsy patients: a 12-year observation," Neurology, vol. 63, no. 6, pp. 1124-1126, 2004.

[15] K. Vonck, V. Thadani, K. Gilbert et al., "Vagus nerve stimulation for refractory epilepsy: a transatlantic experience," Journal of Clinical Neurophysiology, vol. 21, no. 4, pp. 283-289, 2004.

[16] D. Labar, "Vagus nerve stimulation for 1 year in 269 patients on unchanged antiepileptic drugs," Seizure, vol. 13, no. 6, pp. 392398, 2004.

[17] A. V. Alexopoulos, P. Kotagal, T. Loddenkemper, J. Hammel, and W. E. Bingaman, "Long-term results with vagus nerve stimulation in children with pharmacoresistant epilepsy," Seizure, vol. 15, no. 7, pp. 491-503, 2006.

[18] M. Benifla, J. T. Rutka, W. Logan, and E. J. Donner, "Vagal nerve stimulation for refractory epilepsy in children: indications and experience at the Hospital for Sick Children," Child's Nervous System, vol. 22, no. 8, pp. 1018-1026, 2006.

[19] V. De Herdt, P. Boon, B. Ceulemans et al., "Vagus nerve stimulation for refractory epilepsy: a Belgian multicenter study," European Journal of Paediatric Neurology, vol. 11, no. 5, pp. 261269, 2007.

[20] A. P. Amar, M. L. Apuzzo, and C. Y. Liu, "Vagus nerve stimulation therapy after failed cranial surgery for intractable epilepsy: results from the vagus nerve stimulation therapy patient outcome registry," Neurosurgery, vol. 62, supplement 2, pp. 506-513, 2008.

[21] R. E. Elliott, A. Morsi, S. P. Kalhorn et al., "Vagus nerve stimulation in 436 consecutive patients with treatment-resistant epilepsy: long-term outcomes and predictors of response," Epilepsy and Behavior, vol. 20, no. 1, pp. 57-63, 2011.

[22] R. E. Elliott, A. Morsi, O. Tanweer et al., "Efficacy of vagus nerve stimulation over time: review of 65 consecutive patients with treatment-resistant epilepsy treated with VNS > 10 years," Epilepsy \& Behavior, vol. 20, no. 3, pp. 478-483, 2011.

[23] N. Zamponi, C. Petrelli, C. Passamonti, R. Moavero, and P. Curatolo, "Vagus nerve stimulation for refractory epilepsy in tuberous sclerosis," Pediatric Neurology, vol. 43, no. 1, pp. 2934, 2010.

[24] T. Hallböök, J. Lundgren, K. Stjernqvist, G. Blennow, L.-G. Strömblad, and I. Rosén, "Vagus nerve stimulation in 15 children with therapy resistant epilepsy; its impact on cognition, quality of life, behaviour and mood," Seizure, vol. 14, no. 7, pp. 504-513, 2005.

[25] H. C. Kang, Y. S. Hwang, D. S. Kim, and H. D. Kim, "Vagus nerve stimulation in pediatric intractable epilepsy: a Korean bicentric study," in Advances in Functional and Reparative Neurosurgery, vol. 99 of Acta Neurochirurgica Supplementum, pp. 93-96, Springer, Vienna, Austria, 2006.

[26] E. Rossignol, A. Lortie, T. Thomas et al., "Vagus nerve stimulation in pediatric epileptic syndromes," Seizure, vol. 18, no. 1, pp. 34-37, 2009.

[27] A. Shahwan, C. Bailey, W. Maxiner, and A. S. Harvey, "Vagus nerve stimulation for refractory epilepsy in children: more to VNS than seizure frequency reduction," Epilepsia, vol. 50, no. 5, pp. 1220-1228, 2009.
[28] N. Zamponi, C. Passamonti, E. Cesaroni, R. Trignani, and F. Rychlicki, "Effectiveness of vagal nerve stimulation (VNS) in patients with drop-attacks and different epileptic syndromes," Seizure, vol. 20, no. 6, pp. 468-474, 2011.

[29] E. M. S. Sherman, M. B. Connolly, D. J. Slick, K. L. Eyrl, P. Steinbok, and K. Farrell, "Quality of life and seizure outcome after vagus nerve stimulation in children with intractable epilepsy," Journal of Child Neurology, vol. 23, no. 9, pp. 991-998, 2008.

[30] G. Colicchio, D. Policicchio, G. Barbati et al., "Vagal nerve stimulation for drug-resistant epilepsies in different age, aetiology and duration," Child's Nervous System, vol. 26, no. 6, pp. 811-819, 2010.

[31] B. Majkowska-Zwolińska, P. Zwoliński, M. Roszkowski, and K. Drabik, "Long-term results of vagus nerve stimulation in children and adolescents with drug-resistant epilepsy," Child's Nervous System, vol. 28, no. 4, pp. 621-628, 2012.

[32] M. Wheeler, V. De Herdt, K. Vonck et al., "Efficacy of vagus nerve stimulation for refractory epilepsy among patient subgroups: a re-analysis using the Engel classification," Seizure, vol. 20, no. 4, pp. 331-335, 2011.

[33] R. E. Elliott, S. D. Rodgers, L. Bassani et al., "Vagus nerve stimulation for children with treatment-resistant epilepsy: a consecutive series of 141 cases," Journal of Neurosurgery: Pediatrics, vol. 7, no. 5, pp. 491-500, 2011.

[34] E. A. Pastrana, S. Estronza, and I. J. Sosa, "Vagus nerve stimulation for intractable seizures in children: the university of Puerto Rico experience," Puerto Rico Health Sciences Journal, vol. 30, no. 3, pp. 128-131, 2011.

[35] D. Parain, M. J. Penniello, P. Berquen, T. Delangre, C. Billard, and J. V. Murphy, "Vagal nerve stimulation in tuberous sclerosis complex patients," Pediatric Neurology, vol. 25, no. 3, pp. 213216, 2001.

[36] N. Zamponi, C. Passamonti, S. Cappanera, and C. Petrelli, "Clinical course of young patients with Dravet syndrome after vagal nerve stimulation," European Journal of Paediatric Neurology, vol. 15, no. 1, pp. 8-14, 2011.

[37] D. R. Labar, "Antiepileptic drug use during the first 12 months of vagus nerve stimulation therapy: a registration study," Neurology, vol. 59, pp. 538-543, 2002.

[38] E. García-Navarrete, C. V. Torres, I. Gallego, M. Navas, J. Pastor, and R. G. Sola, "Long-term results of vagal nerve stimulation for adults with medication-resistant epilepsy who have been on unchanged antiepileptic medication," Seizure, vol. 22, no. 1, pp. 9-13, 2013.

[39] S. Chayasirisobhon, L. Cahan, S. H. Choi et al., "Efficacy of neuromodulation therapy with vagus nerve stimulator in patients with drug-resistant epilepsy on unchanged antiepileptic medication regimen for 24 months following the implant," Journal of Neurology \& Neurophysiology, vol. 6, pp. 1-4, 2015.

[40] T. R. Henry, "Anatomical, experimental, and mechanistic investigations," in Vagus Nerve Stimulation, S. C. Schachter and D. Schmidt, Eds., pp. 1-29, Martin Dunitz, London, UK, 2001.

[41] S. G. Gurbani, M. Mittal, N. Gurbani, S. Koulouris, S. Choi, and S. Chayasirisobhon, "Efficacy of rapid cycling of vagus nerve stimulation in pharmaco-resistant epilepsy," Neurology Asia, vol. 9, supplement 1, p. 131, 2004. 


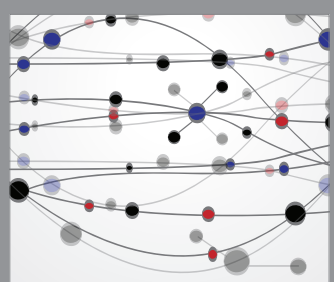

The Scientific World Journal
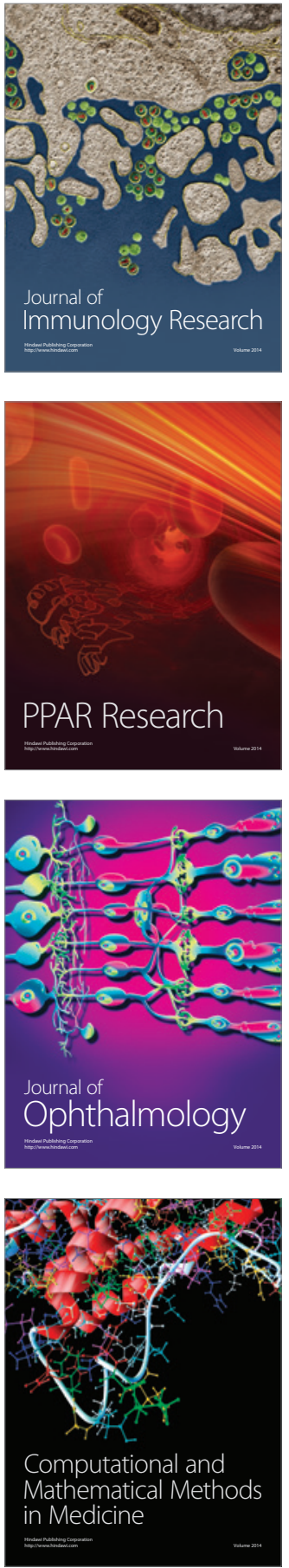

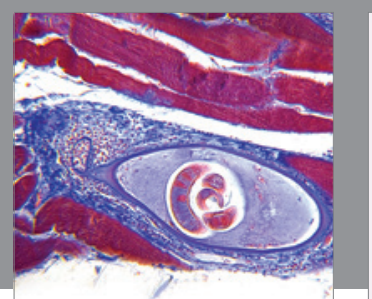

Gastroenterology Research and Practice

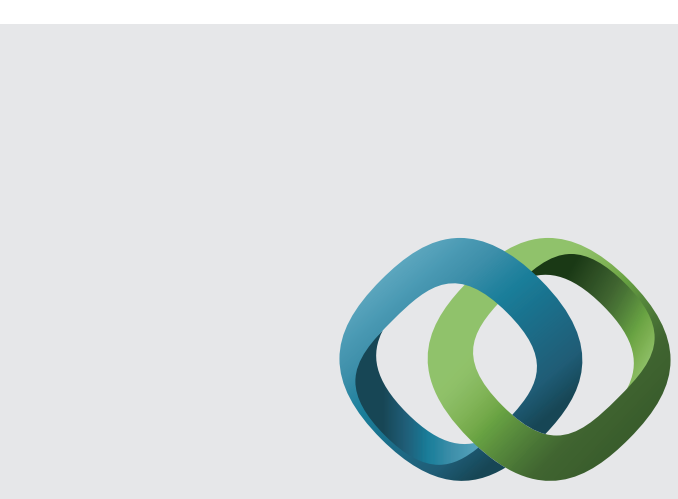

\section{Hindawi}

Submit your manuscripts at

http://www.hindawi.com
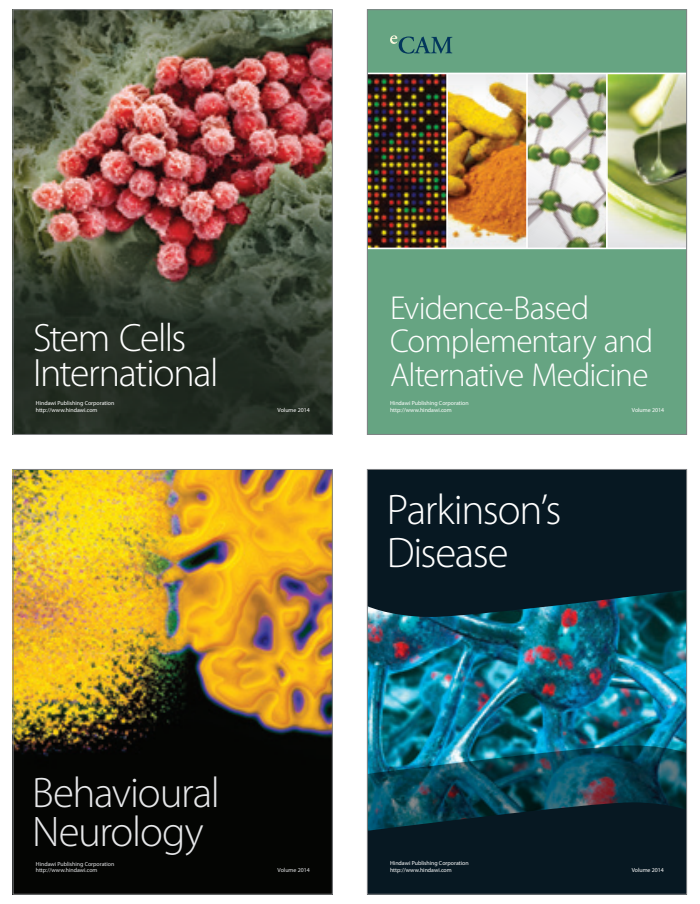
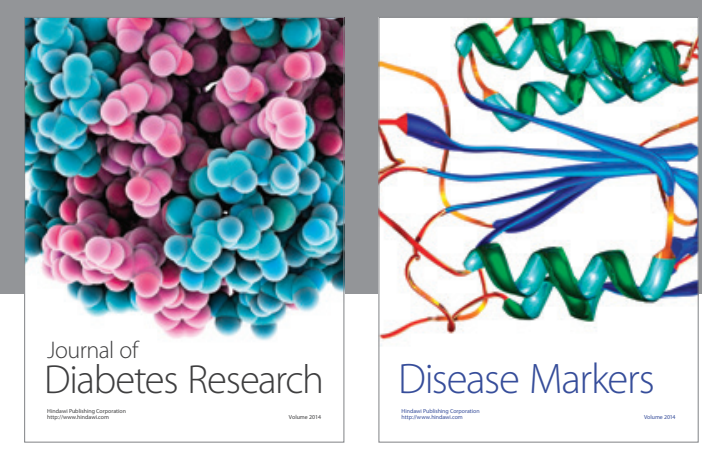

Disease Markers
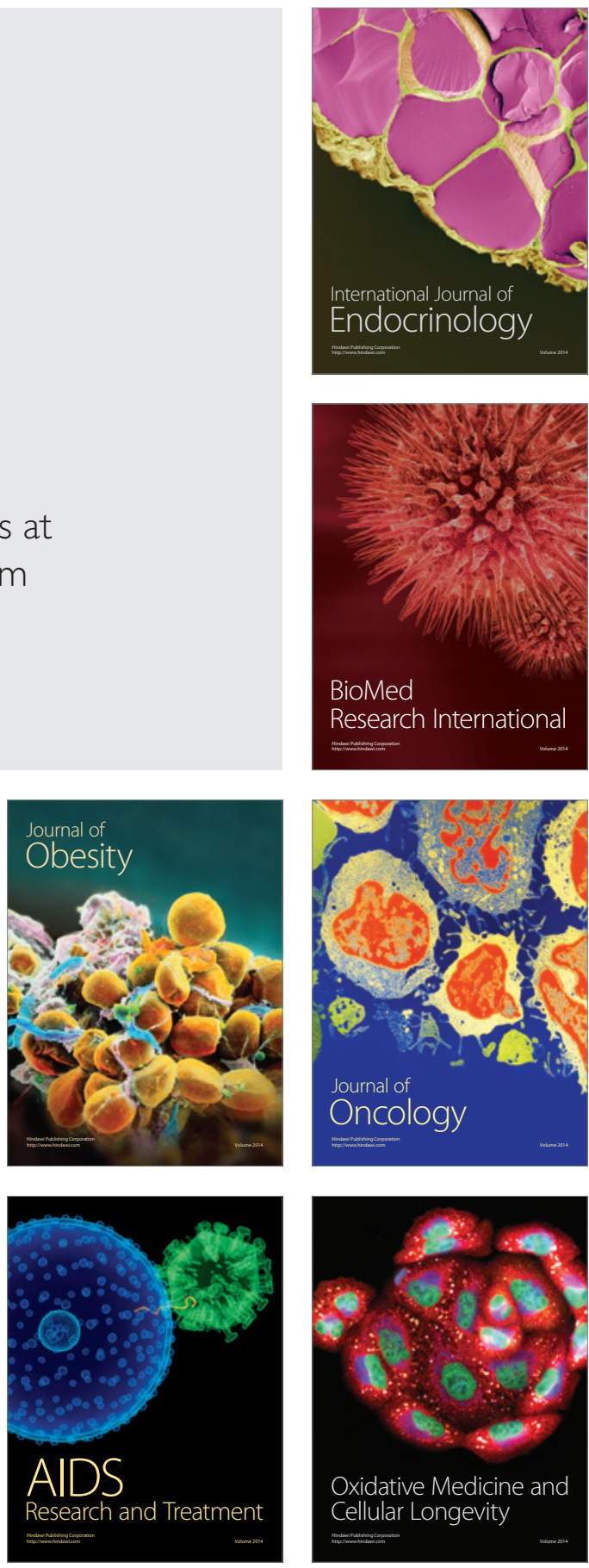